\title{
Dissolving polymer microneedle patches for influenza vaccination
}

\author{
Sean P Sullivan ${ }^{1,4}$, Dimitrios G Koutsonanos ${ }^{2,4}$, Maria del Pilar Martin ${ }^{2}$, Jeong Woo Lee ${ }^{3}$, Vladimir Zarnitsyn ${ }^{3}$, \\ Seong-O Choi ${ }^{3}$, Niren Murthy ${ }^{1}$, Richard W Compans ${ }^{2}$, Ioanna Skountzou ${ }^{2}$ \& Mark R Prausnitz ${ }^{1,3}$
}

Influenza prophylaxis would benefit from a vaccination method enabling simplified logistics and improved immunogenicity without the dangers posed by hypodermic needles. Here we introduce dissolving microneedle patches for influenza vaccination using a simple patch-based system that targets delivery to skin's antigen-presenting cells. Microneedles were fabricated using a biocompatible polymer encapsulating inactivated influenza virus vaccine for insertion and dissolution in the skin within minutes. Microneedle vaccination generated robust antibody and cellular immune responses in mice that provided complete protection against lethal challenge. Compared to conventional intramuscular injection, microneedle vaccination resulted in more efficient lung virus clearance and enhanced cellular recall responses after challenge. These results suggest that dissolving microneedle patches can provide a new technology for simpler and safer vaccination with improved immunogenicity that could facilitate increased vaccination coverage.

The effectiveness of influenza vaccination is limited by the quality and breadth of the immune response and the time required for vaccine delivery ${ }^{1}$. Traditional intramuscular (i.m.) injection requires hypodermic needles that can cause needle phobia and generate biohazardous waste. An advantageous immunization scenario would involve transdermal delivery of the vaccine with a device that promises increased vaccine immunogenicity, enhanced patient compliance via simple self-administration and mass immunization, and elimination of hypodermic needles and their associated biohazardous waste.

This study presents dissolving microneedle patches to increase vaccine immunogenicity by targeting antigen delivery to skin. Microneedles are micron-scale structures that painlessly pierce into the skin to administer vaccines in a minimally invasive and targeted manner ${ }^{2}$. The skin is a highly active immune organ containing a large population of resident antigen-presenting cells ${ }^{3}$. Human clinical studies have shown evidence for dose sparing of intradermal influenza vaccination compared to i.m. immunization, although some other studies have not ${ }^{4-7}$. Intradermal influenza vaccinations at full dose
(15 $\mu \mathrm{g}$ hemagglutinin antigen per strain) and reduced dose $(9 \mu \mathrm{g}$ hemagglutinin per strain) have recently been licensed for human use in some countries (for example, Intanza and IDflu, Sanofi Pasteur). Widespread use of intradermal immunization has been limited by traditional intradermal injections that use the Mantoux technique, which requires specifically trained personnel and is often unreliable ${ }^{8}$. Needle-free transdermal patches have been reported, but the skin's outer layer (stratum corneum) must be disrupted for delivery of large vaccine molecules ${ }^{9}$. In contrast, microneedles are designed to reliably administer antigen at a specific skin depth that maximizes interaction with resident antigen-presenting cells.

Previous studies show that nondissolving metal and silicon microneedle patches can be painless ${ }^{10}$ and can effectively administer vaccine in animals ${ }^{11,12}$, including the influenza vaccine ${ }^{13-15}$. Water-soluble microneedles have been shown to encapsulate bioactive molecules and deliver their cargo into skin ${ }^{16-19}$, but vaccination using this approach has not been studied before.

In this study, we compare standard i.m. immunization to vaccination with polymer microneedles that dissolve within minutes and completely resorb in the skin, resulting in no biohazardous sharps. We show that a single vaccine dose with dissolving microneedles induces protective immune responses superior to those obtained with i.m. injection at the same dose, including increased lung viral clearance. Dissolving microneedles also offer additional benefits, both to the individuals vaccinated and in regard to logistics, including small storage and disposal size, inexpensive fabrication and ease of use to enable self-administration at home.

\section{RESULTS}

Design and fabrication of dissolving polymer microneedles

We designed the polymer material, microneedle geometry and device fabrication process to encapsulate influenza virus while preserving its antigenicity, to insert into skin without mechanical failure and to rapidly dissolve into safe dissolution products. The resulting microneedles measured $650 \mu \mathrm{m}$ tall with sharp tips tapering to a $10-\mu \mathrm{m}$ radius of curvature (Fig. 1a) and were assembled into a multi-needle array (Fig. 1b) that encapsulated $3 \mu \mathrm{g}$ of inactivated influenza virus vaccine per patch.

\footnotetext{
${ }^{1}$ Wallace H. Coulter Department of Biomedical Engineering at Emory University and Georgia Tech, Georgia Institute of Technology, Atlanta, Georgia, USA. ${ }^{2}$ Department of Microbiology \& Immunology and Emory Vaccine Center, Emory University School of Medicine, Atlanta, Georgia, USA. ${ }^{3}$ School of Chemical and Biomolecular Engineering, Georgia Institute of Technology, Atlanta, Georgia, USA. ${ }^{4}$ These authors contributed equally to this work. Correspondence should be addressed to M.R.P. (prausnitz@gatech.edu) or I.S. (iskount@emory.edu).
}

Received 30 July 2009; accepted 23 April 2010; published online 18 July 2010; doi:10.1038/nm.2182 
Figure 1 Dissolving polymer microneedle patches. (a) Side view of dissolving polymer microneedles. (b) Relative height of an array of microneedles next to a US nickel coin. (c) En face view of porcine cadaver skin after insertion and removal of microneedles, showing delivery of the encapsulated compound (sulforhodamine). (d) Fluorescence micrograph of pig skin histological section after insertion of dissolving microneedles ex vivo. (e) Brightfield micrograph of the same skin section with H\&E staining.

We fabricated the microneedles by room-temperature $\left(23^{\circ} \mathrm{C}\right)$ photopolymerization of a liquid monomer (vinyl pyrrolidone) within a microneedle mold to form polyvinylpyrrolidone (PVP) microneedles that encapsulate the lyophilized vaccine. This process avoids the need for organic solvents or elevated temperatures that can damage vaccine or other biomolecule stability. We chose PVP as the structural material for the polymer microneedles used in this study because it is biocompatible, mechanically strong and highly water soluble ${ }^{20}$.

\section{Insertion and dissolution of microneedles in skin}

The resulting microneedles were able to be inserted into porcine skin with gentle force applied by the thumb (Fig. 1c). We determined the fracture force of the microneedles to be $0.13 \pm 0.03 \mathrm{~N}$ per needle, which provides a twofold margin of safety over the force $(0.058 \mathrm{~N}$ per needle $)$ required for insertion into skin using microneedles of this geometry, according to previous measurements ${ }^{21}$. Upon insertion into porcine cadaver skin, microneedles penetrated to a depth of approximately $200 \mu \mathrm{m}$ and deposited their encapsulated payload largely within the epidermis (Fig. 1d,e). This localization is likely to be similar in human skin, which has comparable thickness to porcine $\operatorname{skin}^{22}$.

To characterize the kinetics of dissolution in skin, we inserted microneedles into porcine skin and monitored them over time. Significant dissolution occurred within $1 \mathrm{~min}$, and after $5 \mathrm{~min}$ the microneedles were $89 \pm 3 \%$ (by mass) dissolved (Fig. 2a). Given the similarity of porcine and human skin, we expect that microneedle dissolution in human skin could also be complete within just a few minutes. Because we used mouse skin for the in vivo vaccination experiments described below, we also measured the dissolution kinetics of dissolving microneedles encapsulating the viral antigen in mice. In this scenario, microneedle dissolution was slower but nonetheless increased with time $(P<0.05)$, depositing $34 \pm 17 \%, 63 \pm 10 \%$ and

a

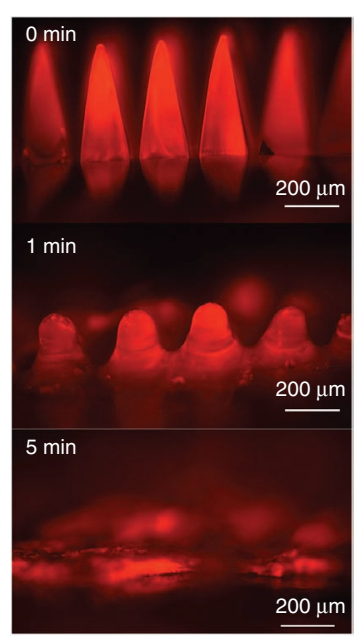

b
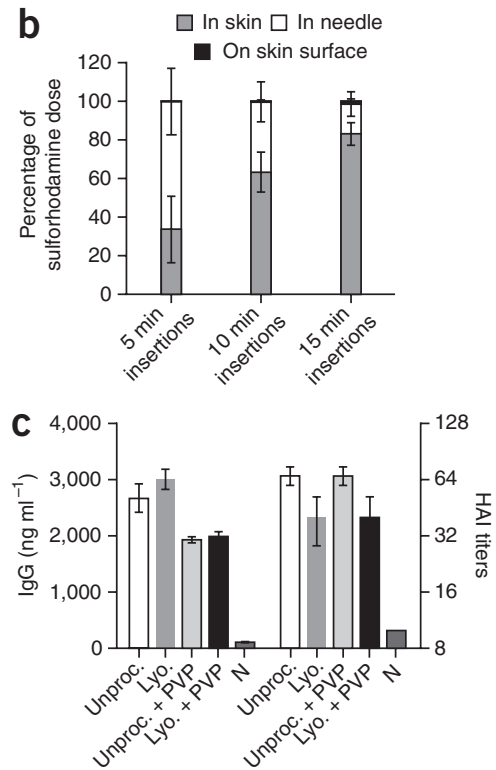
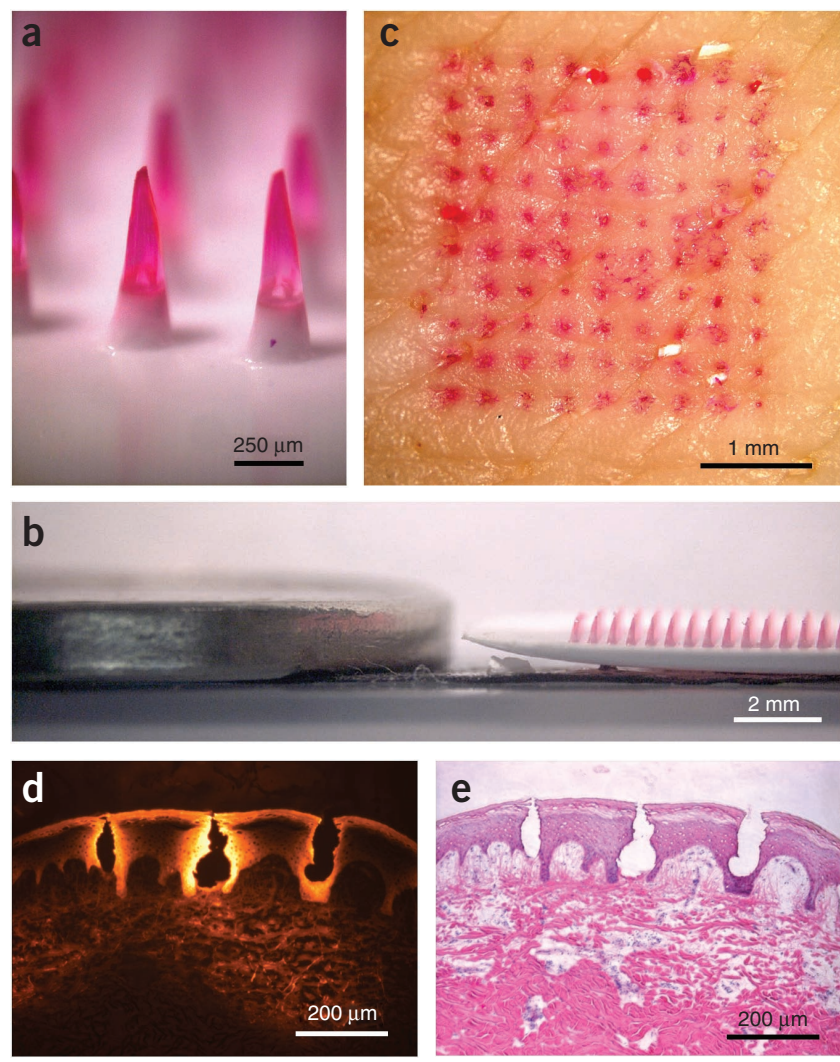

$83 \pm 6 \%$ of the polymer in the skin after 5,10 and $15 \mathrm{~min}$, respectively, and leaving almost no residue on the skin surface (Fig. 2 b).

\section{Antigen stability}

To assess the stability of the inactivated influenza vaccine in dissolving microneedles, we identified two steps during the fabrication of PVP microneedles that might cause damage: the initial lyophilization of vaccine and the subsequent encapsulation within microneedles during polymerization.

To analyze the individual effects of lyophilization and PVP, we administered inactivated influenza virus i.m. in mice as the original vaccine solution, after lyophilization, as the original vaccine solution mixed with PVP and after lyophilization and encapsulation within PVP microneedles. Compared to naive mice, all four vaccinated groups showed elevated influenza-specific IgG titers and hemagglutination

Figure 2 Delivery to skin using microneedles. (a) Polymer microneedle dissolution in pig skin ex vivo. Top, before insertion; middle, remaining polymer 1 min after insertion in skin; bottom, remaining polymer 5 min after insertion in skin. (b) Dissolving microneedle delivery efficiency to mice in vivo. Sulforhodamine was encapsulated within microneedles and administered to mice ( $n=5$ for each time point). The delivery efficiencies for the three time points were statistically different from one another (Student's $t$ test, $P<0.05$ ). (c) Effect of PVP and lyophilization on vaccine immunogenicity. Mice $(n=3)$ were immunized i.m. with $20 \mu \mathrm{g}$ inactivated influenza virus (A/PR/8/34) that was either lyophilized or in solution with or without PVP added. Serum IgG antibody titers and $\mathrm{HAl}$ were measured $14 \mathrm{~d}$ after immunization. Unproc., unprocessed inactivated influenza virus in PBS; Lyo., lyophilized inactivated influenza virus redissolved in PBS; Unproc. + PVP, unprocessed inactivated influenza virus in PBS mixed with PVP; Lyo. + PVP, lyophilized inactivated influenza virus encapsulated in PVP; N, naïve mice. Error bars represent s.d. from three to five independent experiments. 

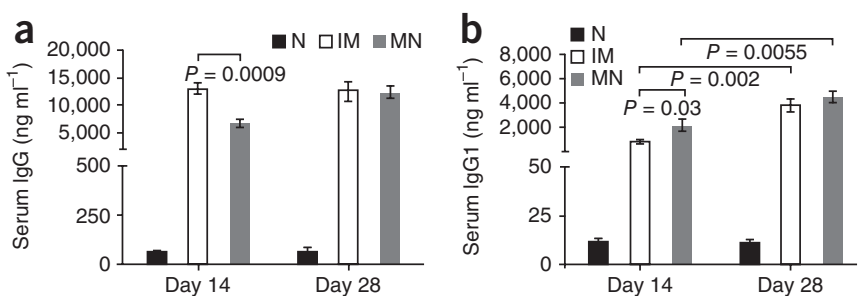

Figure 3 Microneedle immunization studies. (a) Serum influenza-specific IgG titers 14 and $28 \mathrm{~d}$ after immunization. Mice $(n=12)$ were immunized i.m. with inactivated influenza virus (A/PR/8/34) or via a microneedle patch encapsulating the same amount of virus. (b-d) IgG1 titers (b), IgG2a titers (c) and HAI titers (d) on days 14 and 28. (e) Survival rates of immunized and naive mice upon lethal challenge with five times the $L_{50}$ of homologous influenza virus. (f) Percentage of body weight changes upon lethal challenge. $\mathrm{N}$, naïve group; i.m., intramuscularly immunized group; MN, microneedle-immunized group; Inf., unimmunized challenged group. Data shown are means \pm s.e.m. HAl titers are depicted as geometric mean titers (GMT) with 95\% confidence interval (Cl).
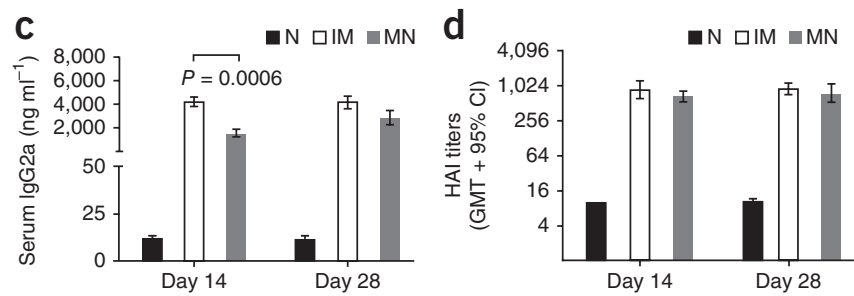

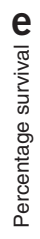
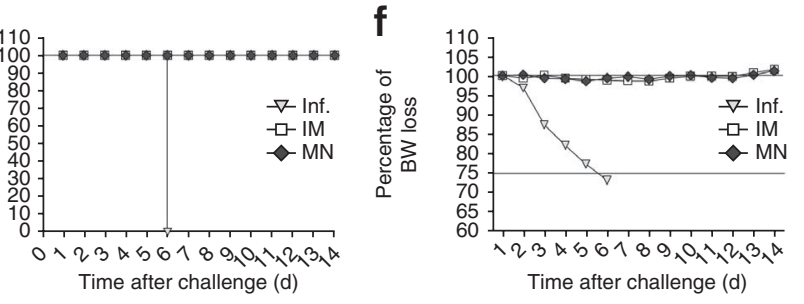

inhibition (HAI) titers (Fig. 2c, $P<0.01$ ). Among the four vaccinated groups, there was no significant effect of vaccine processing or formulation on IgG or HAI titers $(P>0.05)$.

\section{Humoral immune responses}

The efficacy of skin immunization with dissolving microneedles was determined in BALB/c mice that received a single dose of $6 \mu \mathrm{g}$ of whole, encapsulated, inactivated influenza virus. The microneedle patches were applied on the caudal dorsal area of skin for approximately $15 \mathrm{~min}$, which was sufficient to dissolve the microneedles and deliver at least $80 \%$ of the antigen into skin. We compared induction of humoral immune responses after i.m. immunization, which is the standard influenza vaccination method, with those generated using dissolving microneedles at the same vaccine dose (Fig. 3a-d). We collected blood on days 14 and 28 after immunization to determine the concentrations of influenza-specific antibodies. Mice immunized with microneedles showed slightly lower influenza-specific IgG titers than the i.m. group by day 14 (Fig. 3a, $P<0.0009$ ). Titers were at similar levels for both i.m. and microneedle groups at day $28(P=0.9)$.

We also determined the concentrations of influenza-specific isotypes, IgG1 and IgG2a, at 14 and $28 \mathrm{~d}$ after immunization. At day 14 , microneedle-immunized mice had more pronounced IgG1 titers than the i.m. group (Fig. $\mathbf{3 b}, P=0.03$ ), whereas the i.m.-immunized mice showed significantly stronger IgG2a responses than the microneedle group (Fig. 3c, $P=0.0006$ ). At day 28 there were no significant differences in the isotype levels between the groups. This indicates that the i.m. group had Thelper type $1\left(\mathrm{~T}_{\mathrm{H}} 1\right)$-biased responses early after immunization ( IgG1/IgG2a ratio $=0.2)$, but levels of these isotypes were similar after 1 month $(\operatorname{IgG} 1 / \operatorname{IgG} 2 \mathrm{a}=0.9)$. In contrast, the microneedle group showed a slight predominance of IgG1 production over time (IgG1/IgG2a in the range of 1.35 to 1.53 ) (Fig. 3b,c).

HAI activity is generally used as the serological measure for functional antibodies associated with protection. We observed high HAI titers after one immunization (Fig. 3d). HAI titers detected in the microneedle group were similar to each other on days 14 and 28 and to i.m. group titers too (Fig. 3d), demonstrating that a single microneedle immunization induced high levels of functional antibodies.

\section{Protection against lethal viral challenge}

To determine whether microneedle immunization can confer protective immunity, we challenged the immunized groups with five times the half-maximal lethal dose $\left(\mathrm{LD}_{50}\right)$ of mouse-adapted PR8 influenza virus $30 \mathrm{~d}$ after vaccination. All immunized animals survived challenge (Fig. 3e) and lost $<5 \%$ body weight (Fig. 3f), showing that vaccine delivery with dissolving microneedles provided protection equal to the i.m. group. In contrast, the unimmunized group did not survive beyond $6 \mathrm{~d}$ after challenge (Fig. $3 f$ ).

We then investigated the ability of challenged mice to clear influenza virus from the lung $90 \mathrm{~d}$ after vaccination to assess longevity and efficiency of recall responses. On day 4 after challenge, the i.m.-immunized mice showed a decrease in lung viral titers of a factor of $1 \times 10^{3}$ compared to unimmunized infected mice, whereas microneedle-immunized mice showed a marked decrease in lung viral titers of a factor of $1 \times 10^{6}$ (Fig. 4a). As the challenge of the mice took place three months after vaccination, these findings indicate that microneedle immunization induced more robust recall responses than i.m. vaccination, as shown by more efficient virus clearance.

\section{Recall immune responses}

To evaluate the induction of local immune responses, we measured influenza-specific IgG and IgA titers in lungs of challenged mice $90 \mathrm{~d}$ after immunization. We found that soluble IgA titers were modestly increased in vaccinated groups and were similar among microneedle and i.m. groups (Fig. 4b). Lung IgG titers were also similar in microneedle and i.m.-immunized mice, including IgG1 and IgG2a isotype profiles (Fig. 4c). Systemically, we observed that challenged mice had serum influenza-specific IgG titers similar to those observed $28 \mathrm{~d}$ after immunization, with no significant differences among immunized groups (Fig. 4d). Serum HAI titers were also similar in all immunized challenged groups, consistent with total antibody levels (Fig. 4e). Although we noted an increase in IgG1 titers after infection in vaccinated mice, microneedle-immunized mice had a higher IgG1/IgG2a ratio than the i.m. group, as observed in pre-challenge samples (Fig. 4f). Thus, changes in antibody levels were consistent with protective responses in immunized mice. Overall, these data demonstrate that microneedle vaccination induces similar antibody recall responses compared to i.m. vaccination.

Antibody-secreting cells (ASCs) are partly responsible for recall immune responses that confer protection against influenza infection. We examined mice challenged $90 \mathrm{~d}$ after immunization for influenza IgG ASCs in spleen and lungs on day 4 after infection. In spleen, ASC numbers were elevated in both the microneedle and 

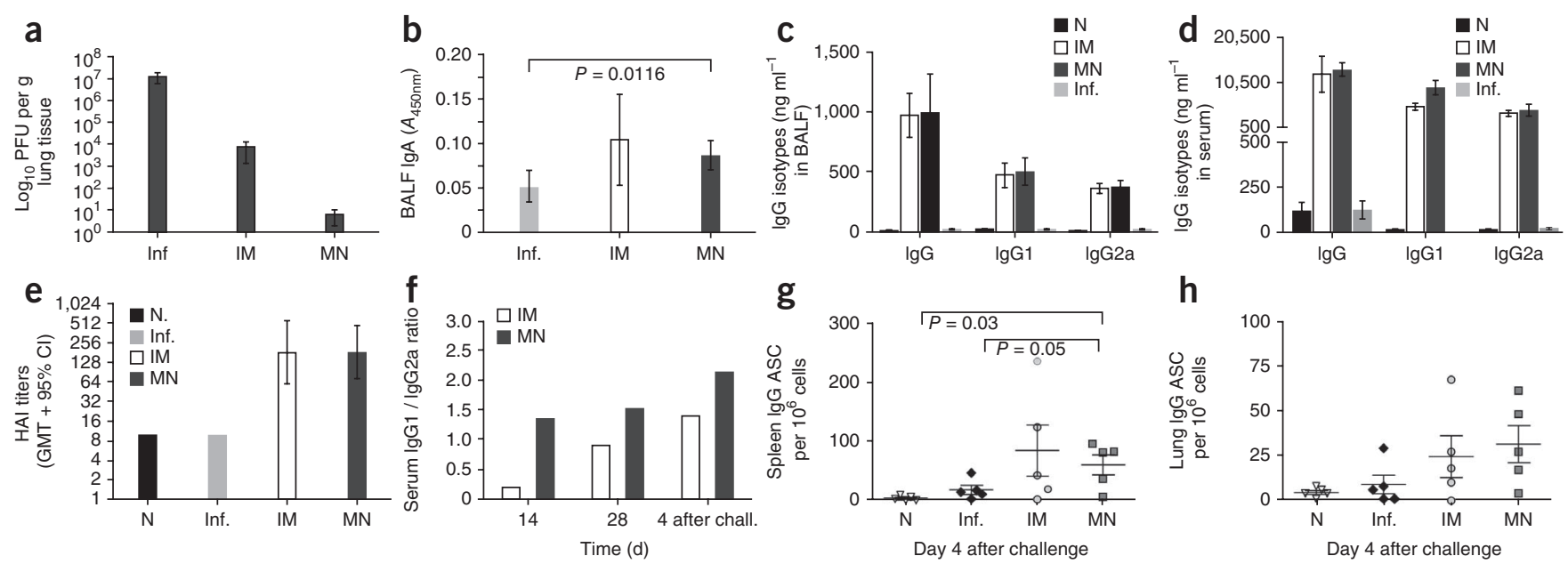

h

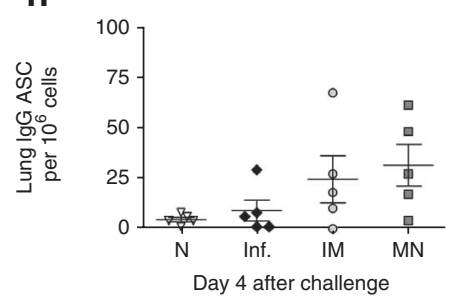

Figure 4 Long-lived immune responses. Mice immunized with influenza virus by microneedle or i.m. route of delivery were challenged with live homologous virus $90 \mathrm{~d}$ after immunization; bronchoalveolar lavage fluid (BALF) and sera were collected at $4 \mathrm{~d}$ after challenge. (a) Lung virus titers (d) plaque assay. PFU, plaque-forming units. (b) Lung IgA titers determined by quantitative ELISA. (c) Lung IgG titers and their isotypes. (d) Serum IgG titers and their isotypes. (e) Serum HAI titers after infection, determined as geometric mean titers (GMT) with 95\% confidence intervals (CI). (f) Serum IgG1/lgG2a ratio on days 14 and 28 after immunization and on day 4 after challenge. (g) Influenza-specific IgG ASCs from splenocytes re-stimulated with inactivated influenza virus. (h) Lung influenza-specific IgG ASCs. Data shown are means \pm s.e.m.

i.m. groups; despite a lack of noticeable differences between groups, the microneedle group was the only one showing significantly higher numbers of ASCs than naive or infected mice (Fig. 4g, $P<0.05$ ). In lungs, we observed that the microneedle and i.m. groups had three to five times higher ASC numbers than unimmunized infected or naive mice (Fig. $4 \mathbf{h}$ ). These results suggest that a skin vaccination route using dissolving microneedles induces sustained humoral immune responses in lungs at least as strong as responses induced by i.m. immunization.

\section{Induction of systemic cytokine responses}

We next investigated induction of cellular immune responses systemically upon challenge $90 \mathrm{~d}$ after immunization. We restimulated splenocytes isolated from challenged mice on day 4 with hemagglutinin major histocompatibility complex (MHC) class I- and hemagglutinin MHC class II-restricted peptides or inactivated influenza virus for $48 \mathrm{~h}$ and $72 \mathrm{~h}$ to determine the contribution of $\mathrm{CD}^{+}$and $\mathrm{CD}^{+}{ }^{+} \mathrm{T}$ lymphocytes secreting interleukin-4 (IL-4) and interferon- $\gamma($ IFN- $\gamma$ ) (Fig. 5a,b). IL-4 secretion was higher in the i.m. group in the presence of class I or class II peptides, although increases were more prominent with class I, suggesting increased $\mathrm{CD}^{+} \mathrm{T}$ cell-derived response (Fig. 5a). In contrast, levels of IFN- $\gamma$ secreted by $\mathrm{CD}^{+}$or $\mathrm{CD} 4^{+}$cells were twoto three-fold higher in the microneedle group when compared to i.m.-injected mice (Fig. 5b). Naive mice did not show any differences in cytokine levels from unimmunized infected mice (data not shown). Elevated IFN- $\gamma$ concentrations in microneedle-immunized mice suggest that microneedle immunization generates strong $\mathrm{T}_{\mathrm{H}} 1$

Figure 5 Cellular immune responses after challenge. Cellular immune responses were determined in splenocyte cultures and lung suspensions. (a) IL-4 concentrations, determined from 72-h splenocyte culture after isolation from immunized and unimmunized mice at $4 \mathrm{~d}$ after challenge and re-stimulation with hemagglutinin (HA) MHC class I and II influenzaspecific peptides. (b) IFN- $\gamma$ concentrations in 72-h splenocyte cultures. (c) Lung IL-21 concentrations. (d) Lung IFN- $\gamma$ concentrations. (e) Lung TNF- $\alpha$ concentrations. (f) Lung IL-12 p70 concentrations. Data shown are means \pm s.e.m. and effector responses, which are necessary to support cytotoxic activity, events that are crucial for viral clearance ${ }^{23}$.

\section{Assessment of cellular immune responses in lungs}

To assess cellular immune responses elicited in the mucosal compartment, we re-stimulated lung cell suspensions in vitro with inactivated $\mathrm{A} / \mathrm{PR} / 8 / 34$ influenza virus and assessed the amounts of IL-21, IFN- $\gamma$, tumor necrosis factor- $\alpha$ (TNF- $\alpha$ ), and IL-12 p70. IL-21 is a pleiotropic cytokine known to upregulate genes associated with innate immunity and $\mathrm{T}_{\mathrm{H}} 1$ responses ${ }^{24}$, as well as regulating $\mathrm{B}$ cell isotype
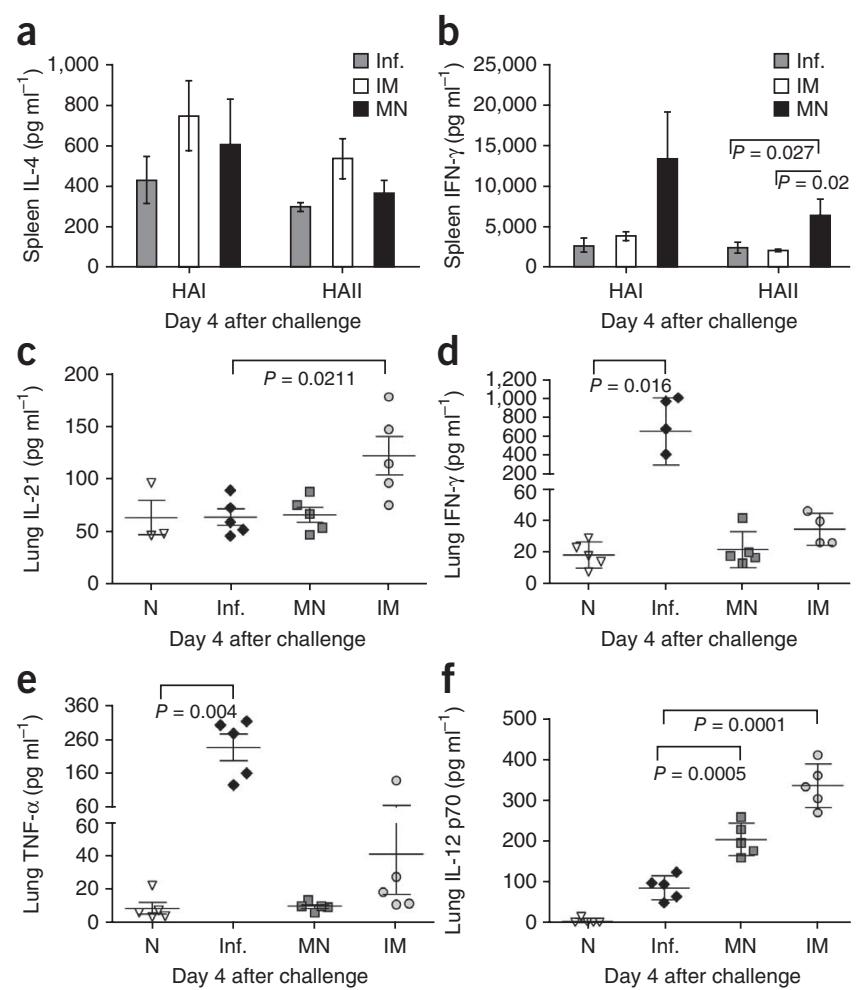
class switching ${ }^{25}$. It also augments IFN- $\gamma$ production in vitro when combined with other cytokines ${ }^{26}$. We found that IL-21 amounts in lungs of i.m.-vaccinated mice were significantly higher than in other groups (Fig. 5c, $P=0.0211$ ), with IFN- $\gamma$ production correspondingly upregulated in the same group (Fig. 5d). Unimmunized infected mice showed the highest IFN- $\gamma$ and TNF- $\alpha$ concentrations (Fig. 5d,e), consistent with stronger inflammatory reaction in mice not protected by vaccination. Notably, both i.m. $(P<0.0001)$ and microneedle $(P<0.0005)$ groups had significantly higher IL-12 p70 production than naive or infected groups, which correlates with the high INF- $\gamma$, which was more prominent in the i.m. group (Fig. 5f).

Expression of IFN- $\gamma$, IL-12 p70 and IL-21 induced after polyclonal re-stimulation in lung was higher in the i.m. compared to microneedle group, which suggests stronger local $\mathrm{T}_{\mathrm{H}} 1$ response in the microneedle group upon challenge. In contrast, influenza virus-specific MHC class I- and class II-restricted T cell responses were increased in the spleen of microneedle-immunized groups, indicative of increased recall $\mathrm{CD}^{+}$and $\mathrm{CD} 8^{+} \mathrm{T}$ cell responses systemically. The higher virusspecific IFN- $\gamma$ production in the microneedle-immunized group may reflect enhanced generation and maintenance of memory $\mathrm{T}$ cells that are responsible for the increased virus clearance observed in lungs when compared to the i.m. group. Overall, these data demonstrate that microneedle immunization can generate a robust cellular and humoral immune response similar to that observed with the conventional i.m. route, and they suggest that microneedle immunization can establish a sustained and broader immune response.

\section{Comparison of dissolving polymer and metal microneedles}

As a final set of experiments, we compared the dissolving polymer microneedles used in this study to coated metal microneedles described previously ${ }^{13-15}$ by vaccinating mice with each of these microneedle technologies and measuring humoral and cellular immune responses after two weeks (Supplementary Data). Humoral immune responses were similar (Supplementary Fig. 1), but cellular responses differed (Supplementary Figs. 2 and 3), most notably shown through increased IL- 4 and IFN- $\gamma$ production from inguinal lymph node cells in response to inactivated influenza virus stimulation in mice vaccinated with dissolving polymer microneedles compared to coated metal microneedles. This result suggests that dissolving microneedles not only offer advantages over i.m. injection but may also represent an improvement over coated metal microneedles.

\section{DISCUSSION}

This study aimed to evaluate use of a simple patch-based vaccination method designed to overcome the limitations of hypodermic needle injection, both in terms of targeting skin antigen-presenting cells and avoiding hypodermic needles ${ }^{27,28}$. We therefore designed, fabricated and analyzed a novel dissolving microneedle patch for skin vaccination. Because microneedles dissolve in skin's interstitial fluid, there is no sharps waste, which makes dissolving microneedles impossible to reuse and thereby eliminates the risks of biohazardous sharps.

This new approach incorporates vaccine in a lyophilized form within the structural polymer material of the microneedle, thereby avoiding the need for reconstitution before administration. These polymer microneedles dissolve in the skin within minutes and are safely eliminated by the body, as evidenced by the historical use of PVP as a plasma expander ${ }^{29}$. The use of needles measuring just hundreds of microns in length not only eliminates pain ${ }^{10}$ and enables simple delivery through a thin patch, but also inherently targets antigen to the abundant antigen-presenting cells of skin's epidermis and dermis ${ }^{3}$.
This study demonstrates that influenza vaccine delivery with dissolving microneedles can induce robust humoral and cellular immune responses after a single immunization with a low antigen dose that confers protective immunity against lethal viral challenge. Immunologic responses to microneedle vaccination were similar to those achieved by i.m. injection by some measures and were stronger by others. Overall, microneedle immunization yielded enhanced recall cellular immune responses, increased numbers of antibody-secreting cells and, notably, more efficient viral clearance.

Although it is possible that dissolving microneedles have strong immunogenicity because of an adjuvant effect caused by PVP, we believe that this is unlikely, because i.m. injection of inactivated virus with PVP did not enhance immune response compared to vaccination without PVP. It is also possible that skin flora are drawn into the skin during microneedle insertion and thereby serve as an adjuvant. We think this is also unlikely, as we carefully cleaned the skin before microneedle insertion and because hypodermic needle insertion for i.m. injection could similarly draw in skin flora.

Thus, dissolving microneedle patches may provide not only practical advantages compared to hypodermic needles but also better protective immunity. Similar reports in human studies have shown that intradermal immunization can induce primary immune responses that are equivalent to or surpass i.m. delivery of seasonal influenza vaccine, with possible dose-sparing effects ${ }^{4-7}$. Although this study did not assess dose sparing, the key immunologic difference between vaccine delivery through dissolving microneedles versus i.m. immunization is the 1,000 -fold more efficient lung virus clearance after microneedle vaccination, which is expected to correlate with reduced morbidity and mortality. Of note, we observed this difference upon challenge 3 months after immunization, suggesting that microneedle immunization induces more robust recall immune responses.

These results may be due to higher numbers of antibody-secreting cells found in spleen and lungs of microneedle-immunized mice as well as enhanced cellular memory responses in spleens, as shown by increased IFN- $\gamma$ secretion after in vitro re-stimulation. Cellular immune responses may promote rapid viral clearance from lung and thereby decrease morbidity, for example, via preexisting $\mathrm{CD}^{+}$ $\mathrm{T}$ cell-mediated immunity directed at peptides from conserved internal proteins of the influenza A virus ${ }^{30}$. The enhanced production of serum IgG1 antibodies after microneedle vaccination may also reflect the role of humoral immune responses that assist in effective virus clearance. These differences are probably due to the route of immunization, although antigen formulation, slower release kinetics and other features of the dissolving microneedle delivery system may also have a role.

Immunization via skin may target innate dendritic cell populations directly through lymphatics from proximal draining lymph nodes and simultaneously by activating the rich dendritic cell network that resides in skin. It is well established that the innate immune system has a pivotal role in adaptive immune responses ${ }^{31}$, possibly accounting for the differences we observe between dissolving microneedle patches and i.m. vaccination ${ }^{32,33}$. The early virus clearance from lungs that we observed may be the result of enhanced involvement and mobilization of innate and adaptive cell populations that induce broader humoral and cellular immune responses.

Overall, these results show that dissolving microneedle patches offer an attractive approach to administer influenza vaccine with improved safety, immunogenicity and logistical operations that may enable an increased population coverage for influenza vaccination. The dissolving microneedle vaccine patch developed in this study 


\section{TECHNICAL REPORTS}

also provides a new platform technology for simple administration of other vaccines and medicines to skin without the need for hypodermic needles.

\section{METHODS}

Methods and any associated references are available in the online version of the paper at http://www.nature.com/naturemedicine/.

Note: Supplementary information is available on the Nature Medicine website.

\section{ACKNOWLEDGMENTS}

This study was carried out at the Emory Vaccine Center and the Georgia Tech Center for Drug Design, Development and Delivery and Institute for Bioengineering and Biosciences. The work was supported in part by US National Institutes of Health grants R01-EB006369 and U01-AI084579 and contract HHSN266200700006C. S.P.S. was a trainee supported by a fellowship from the US Department of Education Graduate Assistance in Areas of National Need program. M.d.P.M. was a trainee supported by contract HHSN266200700006C from the US National Institutes of Health-National Institute of Allergy and Infectious Diseases.

\section{AUTHOR CONTRIBUTIONS}

S.P.S., D.G.K., M.d.P.M. and I.S. carried out most experimental studies; J.W.L. and V.Z. prepared microneedles and helped generate the Supplementary Data; S.-O.C. prepared the molds used to fabricate microneedles; S.P.S., D.G.K., I.S. and M.R.P. designed the study and its analysis; S.P.S., I.S. and M.R.P. wrote the manuscript; and N.M., R.W.C., I.S. and M.R.P. supervised the project.

\section{COMPETING FINANCIAL INTERESTS}

The authors declare competing financial interests: details accompany the full-text HTML version of the paper at http://www.nature.com/naturemedicine/.

Published online at http://www.nature.com/naturemedicine/.

Reprints and permissions information is available online at http://npg.nature.com/ reprintsandpermissions/.

1. Centers for Disease Control and Prevention. Influenza activity-United States and worldwide, 2007-08 season. MMWR Morb. Mortal. Wkly. Rep. 57, 692-697 (2008).

2. Prausnitz, M.R., Mikszta, J.A., Cormier, M. \& Andrianov, A.K. Microneedle-based vaccines. Curr. Top. Microbiol. Immunol. 333, 369-393 (2009).

3. Glenn, G.M. \& Kenney, R.T. Mass vaccination: solutions in the skin. Curr. Top. Microbiol. Immunol. 304, 247-268 (2006).

4. Belshe, R.B. et al. Serum antibody responses after intradermal vaccination against influenza. N. Engl. J. Med. 351, 2286-2294 (2004).

5. Holland, D. et al. Intradermal influenza vaccine administered using a new microinjection system produces superior immunogenicity in elderly adults: a randomized controlled trial. J. Infect. Dis. 198, 650-658 (2008).

6. Van Damme, P. et al. Safety and efficacy of a novel microneedle device for dose sparing intradermal influenza vaccination in healthy adults. Vaccine 27, 454-459 (2009).

7. Hickling, J. \& Jones, R. Intradermal Delivery of Vaccines: A Review of the Literature and the Potential for Development for Use in Low- and Middle-Income Countries. (Program for Appropriate Technology in Health, Ferney Voltaire, France, 2009).

8. Flynn, P.M. et al. Influence of needle gauge in Mantoux skin testing. Chest 106, 1463-1465 (1994).
9. Prausnitz, M.R. \& Langer, R. Transdermal drug delivery. Nat. Biotechnol. 26 , 1261-1268 (2008)

10. Gill, H.S., Denson, D.D., Burris, B.A. \& Prausnitz, M.R. Effect of microneedle design on pain in human volunteers. Clin. J. Pain 24, 585-594 (2008).

11. Mikszta, J.A et al. Improved genetic immunization via micromechanical disruption of skin-barrier function and targeted epidermal delivery. Nat. Med. 8, 415-419 (2002).

12. Widera, G. et al. Effect of delivery parameters on immunization to ovalbumin following intracutaneous administration by a coated microneedle array patch system. Vaccine 24, 1653-1664 (2006).

13. Koutsonanos, D.G. et al. Transdermal influenza immunization with vaccine-coated microneedle arrays. PLoS One 4, e4773 (2009).

14. Zhu, Q. et al. Immunization by vaccine-coated microneedle arrays protects against lethal influenza virus challenge. Proc. Natl. Acad. Sci. USA 106, 7968-7973 (2009).

15. Kim, Y.C. et al. Enhanced memory responses to seasonal H1N1 influenza vaccination of the skin with the use of vaccine-coated microneedles. J. Infect. Dis. 201, 190-198 (2010).

16. Miyano, T. et al. Sugar micro needles as transdermic drug delivery system. Biomed. Microdevices 7, 185-188 (2005).

17. Ito, Y., Yoshimitsu, J., Shiroyama, K., Sugioka, N. \& Takada, K. Self-dissolving microneedles for the percutaneous absorption of EPO in mice. J. Drug Target. 14, 255-261 (2006)

18. Lee, J.W., Park, J.H. \& Prausnitz, M.R. Dissolving microneedles for transdermal drug delivery. Biomaterials 29, 2113-2124 (2008).

19. Sullivan, S.P., Murthy, N. \& Prausnitz, M.R. Minimally invasive protein delivery with rapidly dissolving microneedles. Adv. Mater. 20, 933-938 (2008).

20. Robinson, B.V. PVP: A Critical Review of the Kinetics and Toxicology of Polyvinylpyrrolidone (Povidone). (Lewis Publishers, Chelsea, Michigan, 1990).

21. Park, J.-H., Allen, M.G. \& Prausnitz, M.R. Biodegradable polymer microneedles: fabrication, mechanics and transdermal drug delivery. J. Control. Release 104 51-66 (2005).

22. Bronaugh, R.L., Stewart, R.F. \& Congdon, E.R. Methods for in vitro percutaneous absorption studies II. Animal models for human skin. Toxicol. Appl. Pharmacol. 62, 481-488 (1982)

23. McGill, J. \& Legge, K.L. Cutting edge: contribution of lung-resident $T$ cell proliferation to the overall magnitude of the antigen-specific CD8 $\mathrm{T}$ cell response in the lungs following murine influenza virus infection. J. Immunol. 183 4177-4181 (2009)

24. Strengell, M., Sareneva, T., Foster, D., Julkunen, I. \& Matikainen, S. IL-21 upregulates the expression of genes associated with innate immunity and $T_{H} 1$ response. J. Immunol. 169, 3600-3605 (2002).

25. Ozaki, K. et al. A critical role for IL-21 in regulating immunoglobulin production. Science 298, 1630-1634 (2002).

26. Zeng, R. et al. Synergy of IL-21 and IL-15 in regulating $\mathrm{CD} 8^{+} \mathrm{T}$ cell expansion and function. J. Exp. Med. 201, 139-148 (2005).

27. Miller, M.A. \& Pisani, E. The cost of unsafe injections. Bull. World Health Organ. 77, 808-811 (1999).

28. Mitragotri, S. Immunization without needles. Nat. Rev. Immunol. 5, 905-916 (2005).

29. Ravin, H.A., Seligman, A.M. \& Fine, J. Polyvinyl pyrrolidone as a plasma expander studies on its excretion, distribution and metabolism. N. Engl. J. Med. 247, 921-929 (1952)

30. Doherty, P.C. \& Kelso, A. Toward a broadly protective influenza vaccine. J. Clin Invest. 118, 3273-3275 (2008).

31. Katsikis, P.D., Schoenberger, S.P. \& Pulendran, B. Probing the 'labyrinth' linking the innate and adaptive immune systems. Nat. Immunol. 8, 899-901 (2007).

32. Kupper, T.S. \& Fuhlbrigge, R.C. Immune surveillance in the skin: mechanisms and clinical consequences. Nat. Rev. Immunol. 4, 211-222 (2004).

33. Miller, L.S. \& Modlin, R.L. Toll-like receptors in the skin. Semin. Immunopathol. 29, 15-26 (2007). 


\section{ONLINE METHODS}

Cells and virus stocks. Madin-Darby canine kidney cells (American Type Culture Collection CCL 34, American Type Culture Collection) were maintained in DMEM (Mediatech) containing 10\% FBS (Hyclone, ThermoFisher Scientific). Influenza virus stocks (A/PR/8/34, H1N1) were prepared, purified and inactivated as previously described ${ }^{34}$. Inactivated influenza virus suspensions in PBS were lyophilized using settings based on a prior study ${ }^{35}$ and described in the Supplementary Methods. Hemagglutination activity was determined using chicken red blood cells (LAMPIRE Biological Laboratories) as previously described ${ }^{36}$. The mouse-adapted A/PR/8/34 strain was obtained by eight serial passages in lungs of BALB/c mice. The $\mathrm{LD}_{50}$ was calculated by the Reed-Muench formula ${ }^{37}$, and viral titer was determined by plaque assay ${ }^{34}$.

Polymer microneedle fabrication and encapsulation of influenza vaccine. Dissolving polymer microneedles were created via in situ polymerization of liquid monomer within a microneedle mold, as described previously ${ }^{19}$. Briefly, a microneedle master structure was created via a lens-based, lithographic microfabrication process. A reusable inverse mold was created by pouring polydimethylsiloxane (184 Dow Corning) over the master structure, allowing it to cure overnight, and carefully peeling the resulting mold off the master structure. We then applied $100 \mu \mathrm{l}$ of vinylpyrrolidone monomer $(99 \%$, Sigma-Aldrich), free-radical initiator azobisisobutyronitrile (1.0 mol\%) and inactivated influenza virus $\left(6 \mathrm{mg} \mathrm{ml}^{-1}\right)$ to the mold surface and administered vacuum $(-101 \mathrm{kPa})$ for $1-2$ min to pull the solution into the microneedle mold and form the microneedles. Then, a second mixture of $100 \mu \mathrm{l}$ of vinylpyrrolidone monomer and azobisisobutyronitrile initiator (without vaccine) was applied to the surface of the mold to form the patch backing. Finally, the system was placed under an ultraviolet lamp ( $100 \mathrm{~W}, 300 \mathrm{~nm}$, BLAK RAY) to initiate photopolymerization. After $30 \mathrm{~min}$, the PVP microneedle patch was carefully removed from the mold and stored in a desiccator for up to $30 \mathrm{~d}$.

Antigen stability study. Initial studies were conducted to test the stability of the processed antigen. Four different vaccine preparations were administered i.m., as described below, to assess the effect of microneedle fabrication processes on antigen stability in comparison with naïve mice. For the first two groups, $100 \mu \mathrm{g}$ untreated inactivated influenza virus was resuspended either alone or in combination with $83 \mathrm{mg}$ of PVP in $1.0 \mathrm{ml}$ water. For the third group, $100 \mu \mathrm{g}$ lyophilized inactivated influenza virus was resuspended in $1.0 \mathrm{ml}$ water. For the fourth group, $100 \mu \mathrm{g}$ lyophilized inactivated influenza virus was encapsulated in a microneedle patch containing $83 \mathrm{mg}$ PVP, which was dissolved in $1.0 \mathrm{ml}$ water. Two weeks after immunization, sera were collected and tested for influenza-specific IgG titers, as described below.

Immunizations. Female BALB/c mice (Charles River Laboratory) (11 mice per group, 6-8 weeks old) received a single dose of vaccine by microneedle or i.m. immunization. For microneedle delivery, $2 \mathrm{~d}$ before immunization the mice were anesthetized with a ketamine and xylazine cocktail, the dorsal caudal surface was prepared and hair was removed as previously described ${ }^{34}$. Microneedles were manually inserted into the caudal site of the dorsal surface of the skin, left in place for 15 min and then removed. Immunization with $6 \mu \mathrm{g}$ of vaccine was accomplished by inserting two arrays of microneedles at the same time, each encapsulating $3 \mu \mathrm{g}$ of vaccine. The vaccine dose is reported as the mass of virus protein, which was composed of $~ 30 \%$ hemagglutinin protein. I.m. immunization was carried out by injecting $6 \mu \mathrm{g}$ of the vaccine suspended in $50 \mu \mathrm{l}$ of PBS into the upper quadrant of the gluteal muscle. Mouse studies were approved by the Emory University Institutional Animal Care and Use Committee.

Challenge of mice with influenza virus. To determine survival rates and immune responses after challenge, six mice per group were challenged 1 month after immunization by intranasal instillation of $50 \mu \mathrm{l}(180 \mathrm{PFU})$ of live mouse-adapted $\mathrm{A} / \mathrm{PR} / 8 / 34$ virus and monitored for $14 \mathrm{~d}$. For a control group, we included six unimmunized challenged mice. A weight loss exceeding $25 \%$ was used as the experimental end point, at which mice were killed. The challenged mice were monitored daily for signs of morbidity (body weight changes, fever and hunched posture) and mortality.

Characterization of immune response. As described in the Supplementary Methods, blood was collected 14 and $28 \mathrm{~d}$ after immunization to determine humoral immune responses (total IgG, IgG isotypes and HAI titers). Four days after challenge, blood was collected to determine humoral immune responses; spleens were collected to assay antibody-secreting cells and cytokine expressions levels, and lungs were collected to determine lung virus titers, IgG and IgA titers, antibody-secreting cells and cytokine expression levels.

Statistical analyses. The statistical significance of observed differences was calculated by two-tailed unpaired Student's $t$ test and one-way analysis of variance, including Bonferroni's multiple comparison test. Values were considered significant for $P \leq 0.05$. Unless otherwise stated, data were pooled from at least two independent experiments.

34. Skountzou, I., Quan, F.S., Jacob, J., Compans, R.W. \& Kang, S.M. Transcutaneous immunization with inactivated influenza virus induces protective immune responses. Vaccine 24, 6110-6119 (2006).

35. Amorij, J.P. et al. Rational design of an influenza subunit vaccine powder with sugar glass technology: preventing conformational changes of haemagglutinin during freezing and freeze-drying. Vaccine 25, 6447-6457 (2007).

36. Compans, R.W. Hemagglutination-inhibition: rapid assay for neuraminic acidcontaining viruses. J. Virol. 14, 1307-1309 (1974).

37. Reed, L.J. \& Muench, H. A simple method of estimating fifty per cent endpoints. Am. J. Hyg. 27, 493-497 (1938). 